\title{
Secondary infection in severe and critical COVID-19 patients in China: a multicenter retrospective study
}

\author{
Ling Sang ${ }^{1,2 \#}$, Yin $\mathrm{Xi}^{1 \#}$, Zhimin Lin ${ }^{1 \#}$, Ying Pan ${ }^{1 \#}$, Bin Song ${ }^{3 \#}$, Chang-an $\mathrm{Li}^{4 \#}$, Xia Zheng ${ }^{5}$, Ming Zhong ${ }^{6}$, \\ Li Jiang ${ }^{7}$, Chun Pan ${ }^{8}$, Wei Zhang ${ }^{9}$, Zheng Lv $^{10}$, Jiaan Xia ${ }^{11}$, Nanshan Chen ${ }^{12}$, Wenjuan Wu ${ }^{13}$, Yonghao \\ $\mathrm{Xu}^{1}$, Sibei Chen ${ }^{1}$, Dongdong Liu ${ }^{1}$, Weibo Liang ${ }^{1}$, Xuesong Liu ${ }^{1}$, Xiaoqing Liu ${ }^{1}$, Shiyue $\mathrm{Li}^{1}$, Nanshan \\ Zhong ${ }^{1,2}$, Dan $\mathrm{Ye}^{4}$, Yuanda Xu ${ }^{1 *}$, Nuofu Zhang ${ }^{1 *}$, Dingyu Zhang ${ }^{14,15^{*}}$, Yimin $\mathrm{Li}^{{ }^{1 *}}$
}

${ }^{1}$ State Key Laboratory of Respiratory Diseases, Guangzhou Institute of Respiratory Health, The First Affiliated Hospital of Guangzhou Medical University, Department of Pulmonary and Critical Care Medicine, Guangzhou, China; ${ }^{2}$ Guangzhou Laboratory, Jinyintan Hospital, Wuhan, China; ${ }^{3}$ Department of Tuberculosis and Respiratory Disease, Jinyintan Hospital, Wuhan, China; ${ }^{4}$ Department of Nosocomial Infection Management, The First Affiliated Hospital of Guangzhou Medical University, Guangzhou, China; Department of Critical Care Medicine, The First Affiliated Hospital of Zhejiang University, Hangzhou, China; ${ }^{6}$ Department of Critical Care Medicine, Zhongshan Hospital Fudan University, Shanghai, China; ${ }^{7}$ Department of Critical Care Medicine, Xuanwu Hospital, Capital Medical University, Beijing, China; ${ }^{8}$ Department of Critical Care Medicine, Zhongda Hospital, Southeast University, Nanjing, China; ${ }^{9}$ Emergency Department, 900th Hospital of Joint Service Corps of Chinese PLA, Fuzhou, China; ${ }^{10}$ Department of Critical Care Medicine, Union Hospital, Tongji Medical College, Huazhong University of Science and Technology, Wuhan, China; ${ }^{11}$ Department of Tuberculosis, Wuhan Jinyintan Hospital, Wuhan, China; ${ }^{12}$ Department of Respiratory and Critical Care Medicine Wuhan Jinyintan Hospital, Wuhan, China; ${ }^{13}$ Department of Critical Care Medicine, Wuhan Jinyintan Hospital, Wuhan, China; ${ }^{14}$ Research Center for Translational Medicine, Wuhan Jinyintan Hospital, Wuhan, China; ${ }^{15}$ Joint Laboratory of Infectious Diseases and Health, Wuhan Institute of Virology and Wuhan Jinyintan Hospital, Chinese Academy of Sciences, Wuhan, China

Contributions: (I) Conception and design: Y Li, D Zhang, N Zhang, Y Xu; (II) Administrative support: Y Li; N Zhong, X Liu, S Li, D Ye; (III) Provision of study materials or patients: L Sang, B Song, Y Xi, Y Pan, Z Lin, C Li; (IV) Collection and assembly of data: L Sang, B Song, Y Xi, Y Pan, Z Lin, C Li, M Zhong, L Jiang, C Pan, W Zhang, Z Lv, N Chen, D Liu, W Liang; (V) Data analysis and interpretation: X Zheng, J Xia, W Wu, X Liu, C Li, Y Xu, S Chen; (VI) Manuscript writing: All authors; (VII) Final approval of manuscript: All authors.

"These authors contributed equally to this work.

*These authors contributed equally to this work as senior authors.

Correspondence to: Yimin Li, MD, PHD. State Key Laboratory of Respiratory Diseases, Guangzhou Institute of Respiratory Health, The First Affiliated Hospital of Guangzhou Medical University, Department of Pulmonary and Critical Care Medicine, 151 Yanjiang Road, Guangzhou 510120, China. Email: dryiminli@vip.163.com; Dingyu Zhang, MD, PHD. Wuhan Jinyintan Hospital, Research Center for Translational Medicine, Joint Laboratory of Infectious Diseases and Health, Wuhan Institute of Virology and Wuhan Jinyintan Hospital, Chinese Academy of Sciences, 1 Yintan Road, Dongxihu District, Wuhan 430023, Hubei, China. Email: 1813886398@qq.com; Nuofu Zhang, MD. State Key Laboratory of Respiratory Diseases, Guangzhou Institute of Respiratory Health, The First Affiliated Hospital of Guangzhou Medical University, Department of Respiratory Medicine, 151 Yanjiang Road, Guangzhou 510120, China. Email: nfzhanggird@163.com; Yuanda Xu, MD, PHD. State Key Laboratory of Respiratory Diseases, Guangzhou Institute of Respiratory Health, The First Affiliated Hospital of Guangzhou Medical University, Department of Pulmonary and Critical Care Medicine, 151 Yanjiang Road, Guangzhou 510120, China. Email: xuyuanda@sina.com.

Background: Since 2020 COVID-19 pandemic became an emergent public sanitary incident. The epidemiology data and the impact on prognosis of secondary infection in severe and critical COVID-19 patients in China remained largely unclear.

Methods: We retrospectively reviewed medical records of all adult patients with laboratory-confirmed COVID-19 who were admitted to ICUs from January $18^{\text {th }} 2020$ to April $26^{\text {th }} 2020$ at two hospitals in Wuhan, China and one hospital in Guangzhou, China. We measured the frequency of bacteria and fungi cultured from respiratory tract, blood and other body fluid specimens. The risk factors for and impact of secondary infection on clinical outcomes were also assessed.

Results: Secondary infections were very common $(86.6 \%)$ when patients were admitted to ICU for $>72$ hours. The majority of infections were respiratory, with the most common organisms being Klebsiella 
pneumoniae (24.5\%), Acinetobacter baumannii (21.8\%), Stenotrophomonas maltophilia (9.9\%), Candida albicans (6.8\%), and Pseudomonas spp. (4.8\%). Furthermore, the proportions of multidrug resistant (MDR) bacteria and carbapenem resistant Enterobacteriaceae (CRE) were high. We also found that age $\geq 60$ years and mechanical ventilation $\geq 13$ days independently increased the likelihood of secondary infection. Finally, patients with positive cultures had reduced ventilator free days in 28 days and patients with CRE and/or MDR bacteria positivity showed lower 28-day survival rate.

Conclusions: In a retrospective cohort of severe and critical COVID-19 patients admitted to ICUs in China, the prevalence of secondary infection was high, especially with CRE and MDR bacteria, resulting in poor clinical outcomes.

Keywords: COVID-19; intensive care unit (ICU); secondary infection; carbapenem resistant Enterobacteriaceae (CRE); multidrug resistant Acinetobacter baumannii

Submitted Apr 08, 2021. Accepted for publication Jul 02, 2021.

doi: 10.21037/apm-21-833

View this article at: https://dx.doi.org/10.21037/apm-21-833

\section{Introduction}

Since December 2019, the coronavirus disease 2019 (COVID-19) pandemic has resulted in $127,619,612$ laboratory-confirmed cases and 2,791,953 death cases worldwide up to March 31, 2021 (1). According to existing data, approximately $14-26 \%$ of hospitalized COVID-19 patients have needed treatment in an intensive care unit (ICU) $(2,3)$, and the clinical outcomes of these cases has generally been poor, with the mortality rate reaching $61.5 \%$ within 28 days (4).

Secondary infection, including those caused by bacteria and fungi, may occur during the course of respiratory viral infection. The incidence of secondary infections during the 2009 influenza A (H1N1) pandemic was as high as 23\% (5), and was found to cause poor clinical outcomes in critically ill patients (6). Over the past 20 years, although coronaviruses including severe acute respiratory syndrome coronavirus (SARS-CoV), Middle East respiratory syndrome coronavirus (MERS-CoV), and SARS-CoV-2, all of which mainly attack the lungs, have caused several outbreaks worldwide, the epidemiology of secondary infection during coronavirus epidemics still remains unknown. Only one single center retrospective study demonstrated that the presence of SARS-CoV in the ICU led to changes in the pattern of pathogens detected and increased the acquisition rate of multi-drug resistant Staphylococcus aureus (MRSA) (7). The long course of the disease and the immuno-suppressed state of severe and critical COVID-19 patients place them at high risk of secondary infection (8). Descriptive data found that $64.4 \%$ of hospitalized COVID-19 patients were prescribed antibiotics (9) and this proportion in critically ill patients was as high as $97.5 \%$ (10). Unfortunately, until now the epidemiology of secondary infections in severe and critical COVID-19 patients admitted to the ICU remained largely unclear. Therefore, we performed a retrospective study to evaluate the incidence, risk factors and prognosis of secondary infection in severe and critical COVID-19 patients. We present the following article in accordance with the STROBE reporting checklist (available at https:// dx.doi.org/10.21037/apm-21-833).

\section{Methods}

\section{Patients}

The study was conducted in accordance with the Declaration of Helsinki (as revised in 2013). This study was approved separately by the Independence Ethics Committee of Union Hospital, Tongji Medical College, Huazhong University of Science and Technology (Approval No. 20200307), Medical Ethics Committee of Wuhan Infectious Disease Hospital (Approval No. KY-2020-56.01) and Ethics Committee of the First Affiliated Hospital of Guangzhou Medical University (Approval No. 2020-065). Informed consent was waived due to the retrospective nature of the study.

We retrospectively reviewed medical records of all adult patients (>18 years) with laboratory-confirmed COVID-19 who were admitted to the ICU between January 18th 2020 and April $26^{\text {th }} 2020$ at two hospitals in Wuhan, China (Jinyintan Hospital and Union West Hospital) and one 
hospital in Guangzhou, China (The First Affiliated Hospital of Guangzhou Medical University). All three hospitals were designated hospitals for COVID-19 patients. All patients met the criteria of having severe or critical COVID-19 according to the Chinese guidelines (11). The disease is defined as severe type if the patient met one of the following conditions: respiratory distress, respiratory rate $\geq 30 \mathrm{bpm}$ or $\mathrm{SPO}_{2} \leq 93 \%$ on room air or $\mathrm{PaO}_{2} / \mathrm{FiO}_{2} \leq 300 \mathrm{mmHg}$. The disease in defined as critical type if the patient met one of the following conditions: respiratory failure occurs and requires mechanical ventilation, shock, other organ dysfunction needing intensive care unit monitoring and treatment. The above two types of patients will be included in the analysis.

\section{Data extraction}

The clinical data, including patient demographics, comorbidities, laboratory findings, treatment, pathogen culture results, and clinical outcomes were extracted from the electronic records in each hospital, subsequently crosschecked for data accuracy by two independent intensivists. All data were entered into the computerized database for further statistical analyses.

\section{Study definitions}

The management of each patient in the ICU was decided upon by the attending physicians, and was mainly in accordance with the Chinese guidelines (11). The decision to conduct microbiological cultivation was also based on the judgment of the attending physician. Specimens included sputum for non-intubated patients and endotracheal aspirates or bronchoscopically obtained samples for intubated or tracheotomy patients, blood, urine, pleural effusion and any other samples acquired from suspected sites of infection. Secondary infection was defined as at least one or more positive culture result among a patient's specimens after ICU admission of more than 72 hours duration.

\section{Study outcomes}

The primary outcome measure was the frequency of bacterial and fungal organisms cultured from respiratory tract, blood and other body fluid specimens. We also assessed the risk factors for secondary infection, and the impact of this on clinical outcomes of COVID-19 patients.

\section{Statistical analysis}

Descriptive statistics included frequency analysis (percentages) for categorical variables and means (SD) for normally distributed continuous variables, or medians [interquartile range (IQR)] for skewed data. To test for differences, we used two-sample $t$-tests for continuous variables and the $\chi^{2}$ test for discrete variables. The KaplanMeier method was used to depict the probability of survival and to generate survival curves. Variables identified as significant $(\mathrm{P}<0.2)$ were entered into a multivariate logistic regression model to investigate the relationship between explanatory variables and the occurrence of secondary infection. All analyses were carried out using SPSS version 10 (IBM Corp, Armonk, NY, USA). A two-sided P value of 0.05 was considered to be significant.

\section{Results}

During the study period, a total of 190 patients infected with SARS-CoV-2 who met the severe or critical criteria defined by the Chinese guidelines (11) were enrolled. Of the study cohort, 62 patients $(32.6 \%)$ were female, and the mean age was 62.7 (SD 13.3) years. The majority of patients had underlying comorbidities (140/190, 73.7\%). Eleven patients (5.8\%) were classified as "severe", while 179 patients $(94.2 \%)$ were classified as "critical". However, all of them needed respiratory support upon admission to the ICU. Table 1 describes the patient characteristics in detail.

Among the 190 patients, 1,929 specimens were collected (10.2 specimens per patient on average), and 1,104 positive cultures $(57.2 \%)$ were obtained. Of these positive cultures, $935(935 / 1,104,84.7 \%)$ were from the lower respiratory tract, $131(131 / 1,104,11.9 \%)$ were from blood, and 38 (38/1,104, 3.4\%) were from other body fluid specimens. The distribution of cultures in the three hospitals is shown in Table 2.

Of the study cohort, 165 patients $(86.8 \%)$ had positive cultures (including bacteria and fungi) after ICU admission of more than 72 hours duration. Of these, 150 patients showed positive sputum/lower respiratory tract aspirate cultures, followed by 64 patients showing positive blood cultures, and 24 patients with positive cultures from other sites (Table 3).

Of the 1,104 positive cultures obtained from 165 patients, the most common organisms overall were Klebsiella pneumoniae (24.5\%), Acinetobacter baumannii (21.8\%), Stenotrophomonas maltophilia (9.9\%), Candida albicans (6.8\%), 
Table 1 Baseline patient characteristics

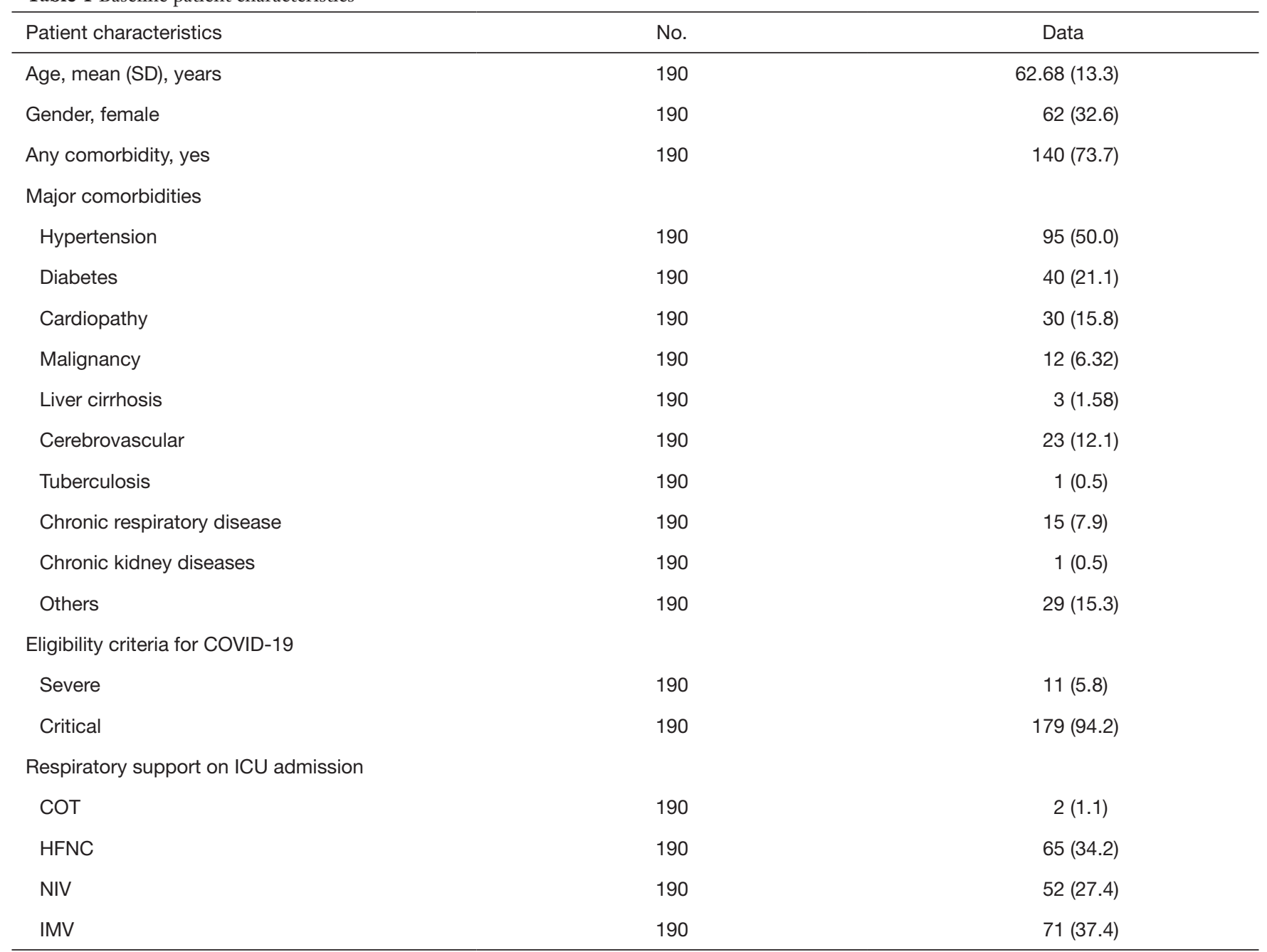

Data are presented as No. (\%) unless otherwise noted. SD, standard deviation; COVID-19, coronavirus disease 2019; ICU, intensive care unit; COT, conventional oxygen therapy; HFNC, high flow nasal cannular; NIV, noninvasive ventilation; IMV, invasive mechanical ventilation.

Table 2 Distribution of cultures in three hospitals

\begin{tabular}{lcccc}
\hline Variable & Total $(\mathrm{n}=190)$ & Jinyintan Hospital $(\mathrm{n}=97)$ & $\begin{array}{c}\text { Union West Hospital } \\
(\mathrm{n}=74)\end{array}$ & $\begin{array}{c}\text { The 1 } 1^{\text {st }} \text { affiliated Hospital of } \\
\text { Guangzhou Medical University }(\mathrm{n}=19)\end{array}$ \\
\hline Inspection frequency & 1,929 & 1,072 & 440 & 417 \\
Positive cultures & $1,104(57.2 \%)$ & $525(49 \%)$ & $335(76.1 \%)$ & $244(58.5 \%)$ \\
From lower respiratory tract & $935(84.7 \%)$ & $414(78.9 \%)$ & $289(86.3 \%)$ & $232(95.1 \%)$ \\
From blood & $131(11.9 \%)$ & $90(17.1 \%)$ & $40(11.9 \%)$ & $1(0.4 \%)$ \\
\#From other body fluid & $38(3.4 \%)$ & $21(4 \%)$ & $6(1.8 \%)$ & $11(4.5 \%)$ \\
specimens & & & & \\
\hline
\end{tabular}

\#Other body fluid specimens included urine, pleural effusion and any other samples acquired from suspected sites of infection. 
Table 3 Characteristics of positive bacterial cultures

\begin{tabular}{|c|c|c|}
\hline Characteristics of positive cultures & No. & Data \\
\hline Number of positive sputum/lower respiratory tract aspirates cultures per patient, median (IQR) & 190 & $3.0(1.0-6.0)$ \\
\hline Frequency of positive sputum/lower respiratory tract aspirates culture among all positive cultures & 150 & \\
\hline 1 positive culture & & $30(20.0)$ \\
\hline$\geq 3$ positive cultures & & $101(67.3)$ \\
\hline Number of positive blood cultures per patient, median (IQR) & 190 & $0(0-1.0)$ \\
\hline Frequency of positive blood culture among all positive cultures & 64 & \\
\hline 1 positive culture & & $32(50.0)$ \\
\hline "Number of other positive cultures per patient, median (IQR) & 190 & $0(0-0)$ \\
\hline Frequency of other positive culture among all positive cultures & 24 & \\
\hline 1 positive culture & & $15(62.5)$ \\
\hline 2 positive cultures & & $5(20.9)$ \\
\hline$\geq 3$ positive cultures & & $4(16.7)$ \\
\hline
\end{tabular}

Data are presented as No. (\%) unless otherwise noted. "Other positive cultures were obtained from urine, pleural effusion and any other samples acquired from suspected sites of infection. ICU, intensive care unit; IQR, interquartile range.

and Pseudomonas spp. (4.8\%). It was noteworthy that the proportions of multidrug resistant (MDR) bacteria and carbapenem resistant Enterobacteriaceae (CRE) were very high $(94.5 \%$ in K. pneumoniae, $98.3 \%$ in A. baumannii and 92.5\% in Pseudomonas spp.). The positive cultures were mainly obtained from sputum and/or lower respiratory tract aspirates. Conversely, only four patients showed six instances of MRSA positivity, all of which were obtained from sputum and/or lower respiratory tract aspirates. More details are shown in Table 4. Of the total positive cultures, 525 were from Jinyintan Hospital (47.6\%), with the most common organisms being $K$. pneumoniae (29.3\%), A. baumannii (22.7\%), C. albicans (10.5\%), Pseudomonas spp. (5.9\%) and S. maltophilia (3.6\%). A total of 335 were from Union West Hospital, with the most common organisms being (36.1\%), K. pneumoniae (32.5\%), S. maltophilia (10.5\%), Pseudomonas spp. (6.6\%) and Escherichia coli (3\%). The remaining 244 positive samples were from The First Affiliated Hospital of Guangzhou Medical University, and the most common organisms were S. maltophilia (22.5\%), Burkholderia cepacia (12.7\%), Ralstonia pickettii (4.9\%), K. pneumoniae (3.3\%) and C. albicans (3.3\%) (Figure 1).

On comparison, patients with positive cultures were older $(63.9 \pm 12.9$ vs. $54.6 \pm 14.1$ years, $\mathrm{P}=0.001)$, had a higher proportion of cardiopathy $(18.2 \%$ vs. $0, \mathrm{P}=0.04)$, and had a longer duration of mechanical ventilation [15.0 (IQR, 7.5-27.0) vs. 4.0 (IQR, 2.5-5.5), $\mathrm{P}=0.03$ ] compared with those without secondary infections. Other baseline characteristics were similar. It seemed that more patients with positive cultures received glucocorticoid exceeding $1 \mathrm{mg} / \mathrm{kg} /$ day for more than 3 days $(73.4 \%$ vs. $54.5 \%$, $\mathrm{P}=0.07)$ and carbapenem for more than 3 days $(49.7 \%$ vs. $33.3 \%, \mathrm{P}=0.16)$ in the week preceding ICU admission. Additionally, more patients with positive cultures received invasive mechanical ventilation upon ICU admission ( $40 \%$ vs. $20 \%, \mathrm{P}=0.05$ ) compared with those patients with negative cultures. However, these differences were not statistically significant (Table 5). Finally, the variables identified as significant $(\mathrm{P}<0.2)$ were entered into a multivariate logistic regression model to investigate the relationship between explanatory variables and the occurrence of secondary infection, and the result showed 
Table 4 Frequency of organisms isolated according to date of acquisition

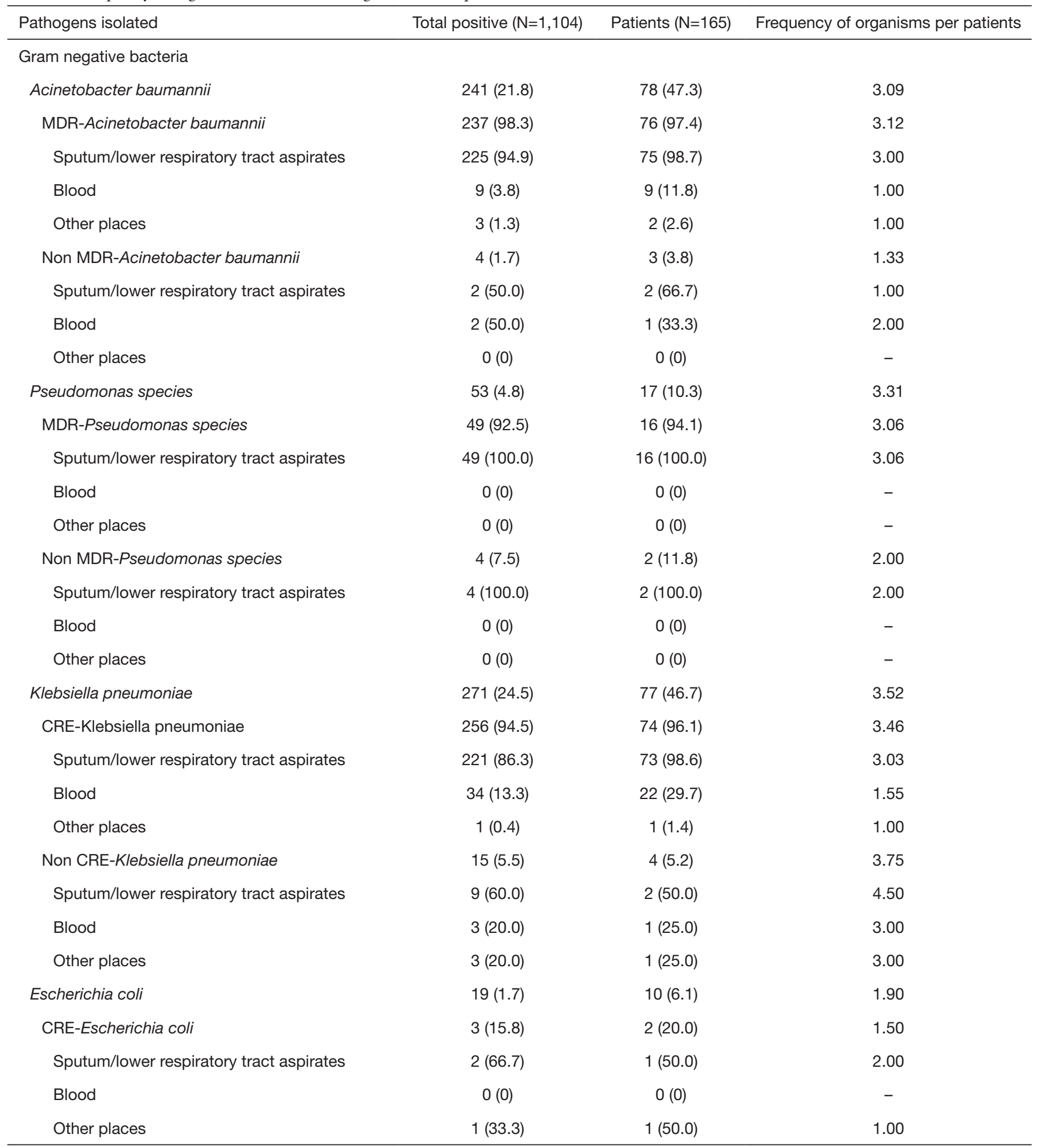

Table 4 (continued) 
Table 4 (continued)

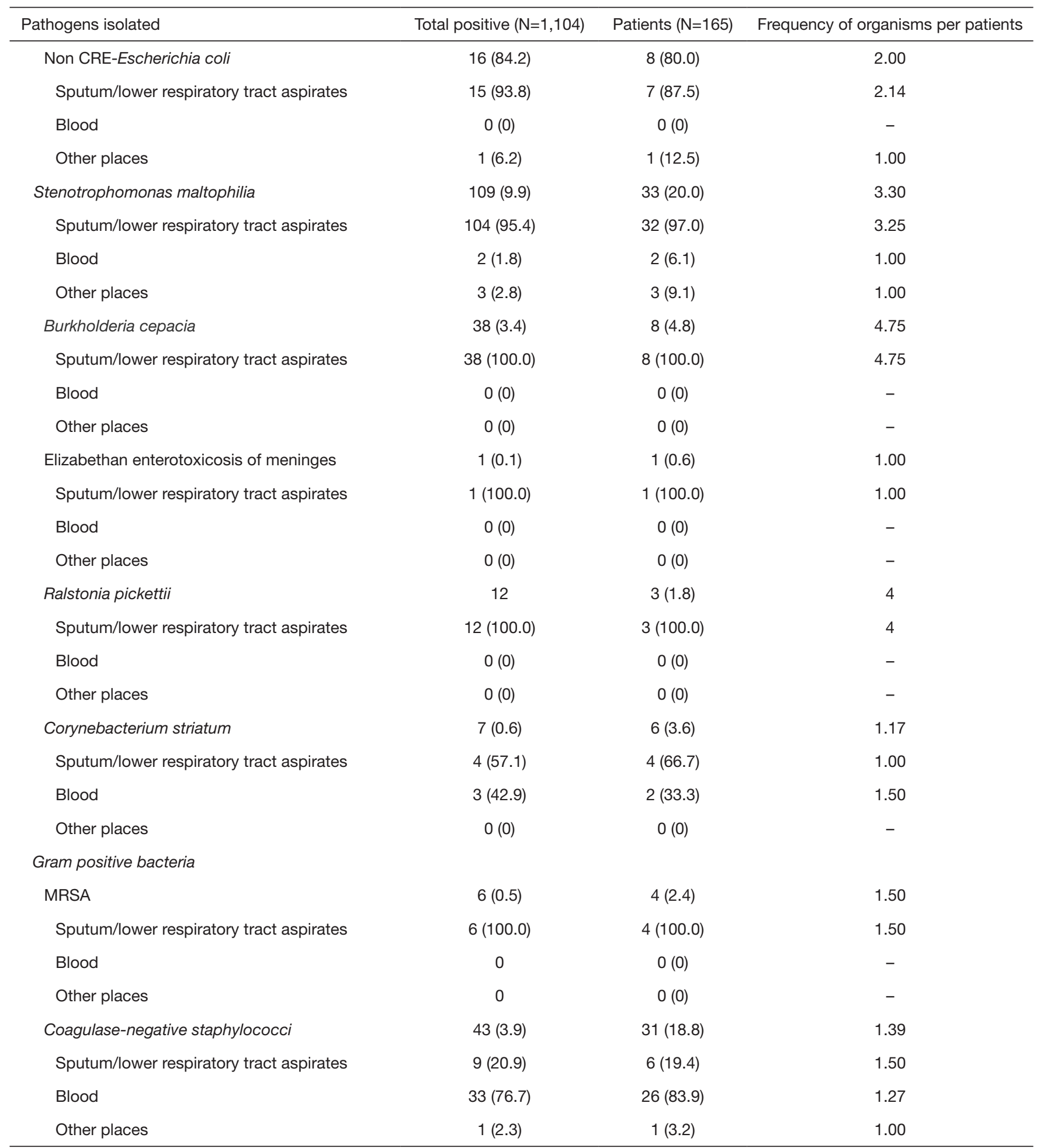

Table 4 (continued) 
Table 4 (continued)

\begin{tabular}{|c|c|c|c|}
\hline Pathogens isolated & Total positive $(\mathrm{N}=1,104)$ & Patients $(\mathrm{N}=165)$ & Frequency of organisms per patients \\
\hline Sputum/lower respiratory tract aspirates & $3(15.0)$ & $2(13.3)$ & 1.50 \\
\hline Blood & $9(45.0)$ & $8(53.3)$ & 1.13 \\
\hline Other places & $8(40.0)$ & $7(46.7)$ & 1.14 \\
\hline Sputum/lower respiratory tract aspirates & $1(33.3)$ & $1(33.3)$ & 1.00 \\
\hline Blood & $1(33.3)$ & $1(33.3)$ & 1.00 \\
\hline Other places & $1(33.3)$ & $1(33.3)$ & 1.00 \\
\hline Enterococcus casseliflavus & $7(0.6)$ & $6(3.6)$ & 1.17 \\
\hline Other places & $0(0)$ & $0(0)$ & - \\
\hline \multicolumn{4}{|l|}{ Fungus } \\
\hline Candida albicans & $75(6.8)$ & $60(36.4)$ & 1.25 \\
\hline Sputum/lower respiratory tract aspirates & $69(92.0)$ & $59(98.3)$ & 1.17 \\
\hline Blood & $5(6.7)$ & $1(1.7)$ & 5.00 \\
\hline Other places & $1(1.3)$ & $1(1.7)$ & 1.00 \\
\hline Non-candida albicans & $52(4.7)$ & $38(23.0)$ & 1.37 \\
\hline Sputum/lower respiratory tract aspirates & $40(76.9)$ & $32(84.2)$ & 1.25 \\
\hline Other places & $0(0)$ & 0 & - \\
\hline
\end{tabular}

Data are presented as No. (\%). MRSA, methicillin-resistant Staphylococcus aureus; CRE, carbapenem resistant Enterobacteriaceae; MDR, multidrug resistant. Other places included urine, pleural effusion and any other samples acquired from suspected sites of infection.

that age $\geq 60$ years [odds ratio (OR) $4.885 ; 95 \%$ confidence interval (CI), 1.813-13.158] and mechanical ventilation $\geq 13$ days (OR 24.759; 95\% CI, 3.044-201.383) independently increased the likelihood of secondary infection in severe and critical COVID-19 patients (Table 6).

Patients with positive cultures had fewer ventilator free days in 28 days compared with those with negative cultures ( $2.2 \pm 5.4$ vs. $6.1 \pm 10.8$ days, $\mathrm{P}=0.02)$, while the mortality at 28 days ICU admission was similar (Table 7, Figure 2). However, when the patients were divided into CRE and/ or MDR bacteria-positive and non-CRE and/or MDR bacteria-positive groups, the patients with CRE and/or MDR bacteria positivity showed lower 28 days survival $(\mathrm{P}=0.02$; Figure 3).

\section{Discussion}

In this work, we evaluate the epidemiology of secondary infections in severe and critical COVID-19 patients. We found that secondary infection was very common $(86.6 \%)$ in 


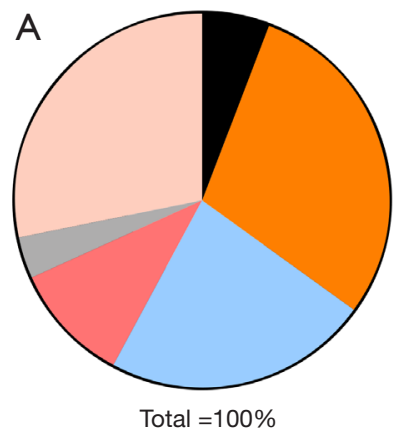

Pseudomonas species Klebsiella pneumoniae Acinetobacter baumannii Candida albicans Stenotrophomonas maltophilia

others
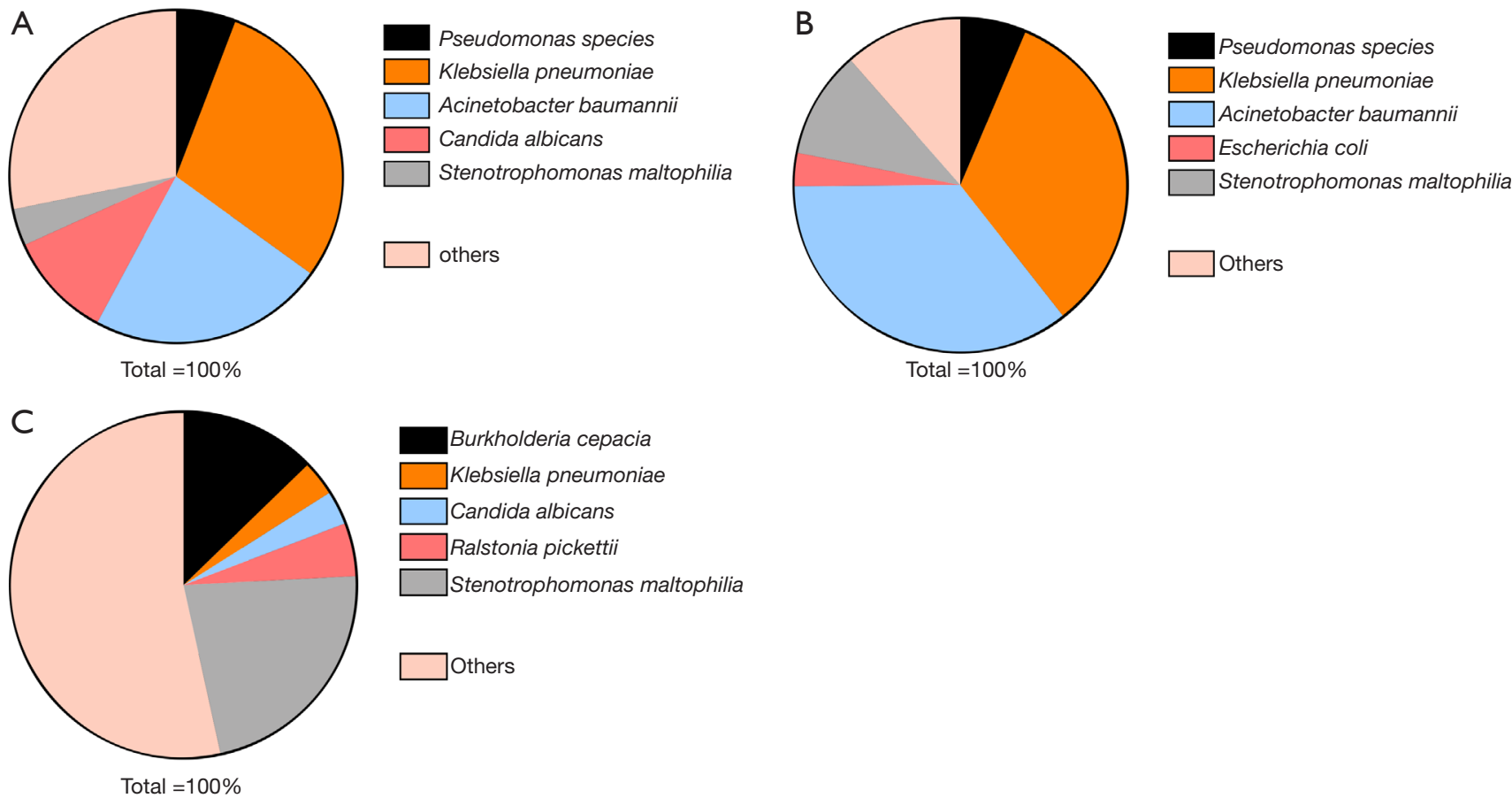

Burkholderia cepacia

Klebsiella pneumoniae

$\square$ Candida albicans

$\square$ Ralstonia pickettii

$\square$ Stenotrophomonas maltophilia

Others

Figure 1 The distribution of organisms isolated in three hospitals. (A) Distribution of organisms isolated in Jinyintan Hospital. (B) Distribution of organisms isolated in Union West Hospital. (C) Distribution of organisms isolated in The First Affiliated Hospital of Guangzhou Medical University.

this patient population when they were admitted to the ICU for more than 72 hours, and the majority were respiratory infections. The most common organisms isolated overall were K. pneumoniae (24.5\%), A. baumannii (21.8\%), S. maltophilia (9.9\%), C. albicans (6.8\%), and Pseudomonas spp. (4.8\%). Furthermore, the proportions of MDR bacteria and CRE were surprisingly high $(94.5 \%$ in K. pneumoniae, $98.3 \%$ in A. baumannii and $92.5 \%$ in Pseudomonas spp.). Interestingly, the distribution of organisms isolated was quite different between Wuhan and Guangzhou; the main organisms isolated in Wuhan were K. pneumoniae and $A$. baumannii, while $S$. maltophilia and $B$. cepacia were more common in Guangzhou. We also found that age $\geq 60$ years and mechanical ventilation $\geq 13$ days (OR 24.759; 95\% CI, 3.044-201.383) independently increased the likelihood of secondary infection in severe and critical COVID-19 patients. Finally, patients with positive cultures had reduced ventilator free days in 28 days and those with CRE and/ or MDR bacteria positivity showed a lower 28-day survival rate.

The coinfection of the SARS-CoV-2 with other microorganisms, which make the diagnosis and treatment more difficult and contribute to the poor prognosis in COVID-19 patients, is crucial in the management of COVID-19 patients (12). However, the true prevalence of coinfection in such patients remained largely unclear. Søgaard et al. observed a high frequency of secondary infections among hospitalized SARS-CoV-2-positive patients. Among 162 SARS-CoV-2-positive hospitalized patients, 31 secondary infections were diagnosed including five viral co-infections, 24 bacterial infections, and three fungal infections, and antibiotic or antifungal treatment was administered in $71(43.8 \%)$ patients. They also found that hospital-acquired bacterial and fungal infections were more frequent among ICU patients than other patients (36.6\% vs. $1.7 \%$ ) (13). However, the prevalence of pulmonary microbial co-infections is modest among COVID-19 patients upon admission to ICU in Region Zealand in Denmark (14).

Secondary infection had always been a common concern in the field of critical care medicine worldwide. In 1995, the results of the EPIC study, which evaluated the prevalence of nosocomial infection in ICUs in Europe, demonstrated that $20.6 \%$ of ICU patients acquired nosocomial infection 
Table 5 Characteristics, treatments, and outcomes of patients with positive cultures compared with those without

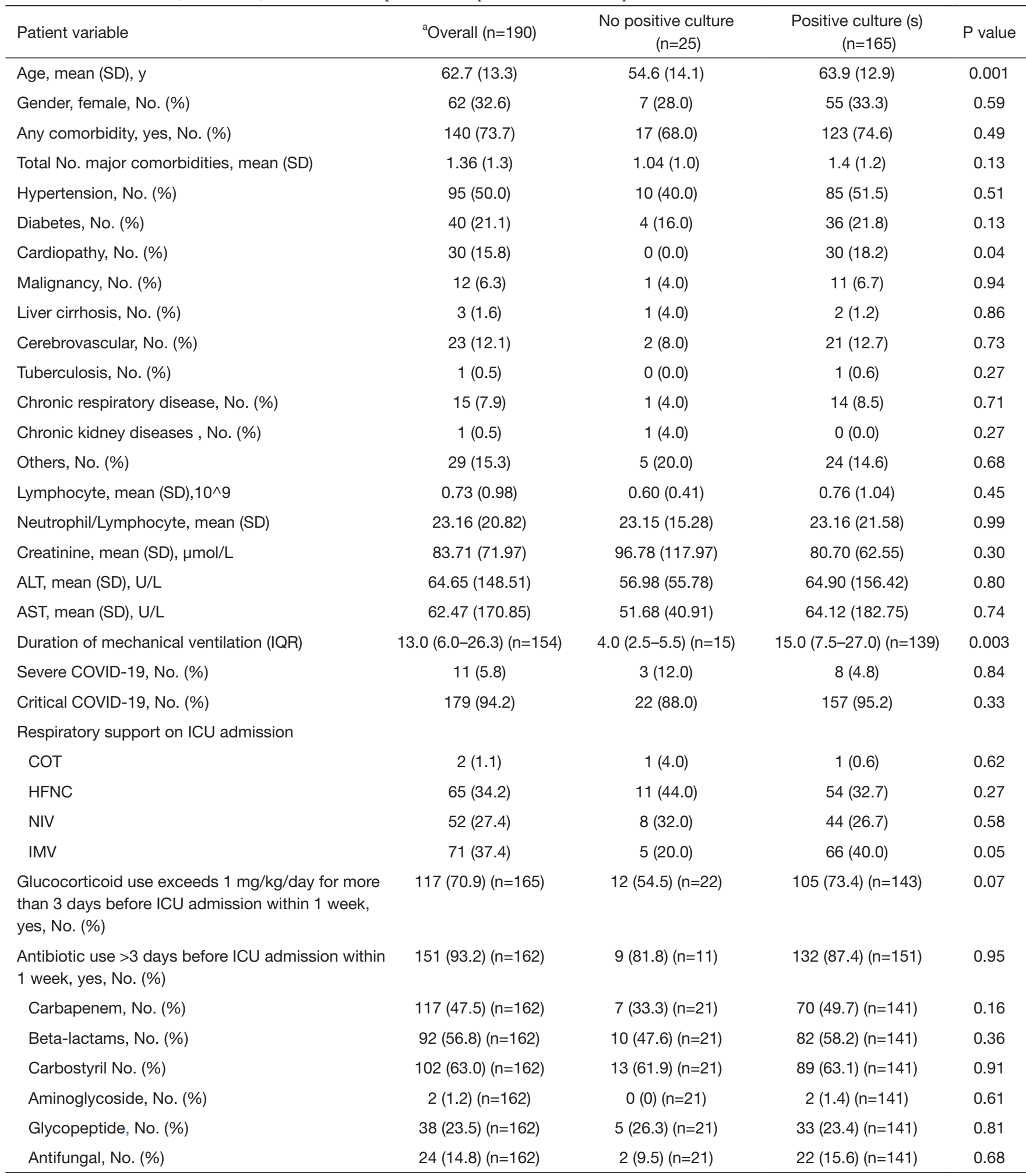

Data are presented as No. (\%) unless otherwise noted. ${ }^{a}$ Total sample size is 190 . Where data are unavailable, sample size for data is provided. SD, standard deviation; IQR, interquartile range; COVID-19, coronavirus disease 2019; ICU, intensive care unit; COT, conventional oxygen therapy; HFNC, high flow nasal cannular; NIV, noninvasive ventilation; IMV, invasive mechanical ventilation. 
Table 6 Independent high risk factors for secondary infection in severe and critical COVID-19 patients upon admission to ICU

\begin{tabular}{lccccc}
\hline Variable & $\mathrm{B}$ & Wald & $\mathrm{P}$ & $\mathrm{OR}$ & $95 \% \mathrm{Cl}$ \\
\hline Age $\geq 60$ years & 1.436 & 9.841 & 0.002 & 4.885 & $1.813-13.158$ \\
Glucocorticoid use exceeds $1 \mathrm{mg} / \mathrm{kg} /$ day for more than 3 days before ICU & 0.808 & 2.155 & 0.142 & 2.244 & $0.763-6.600$ \\
admission within 1 week & & & & \\
IMV & 0.351 & 0.345 & 0.557 & 1.421 & $0.440-4.592$ \\
Carbapenem use $>3$ days before ICU admission within 1 week & 0.054 & 0.009 & 0.926 & 1.056 & $0.339-3.286$ \\
Comorbidity: cadiopathy & 0.568 & 1.223 & 0.269 & 1.764 & $0.645-4.822$ \\
Mechanical ventilation $\geq 13$ days & 3.209 & 9.005 & 0.003 & 24.759 & $3.044-201.383$ \\
\hline
\end{tabular}

OR, odds ratio; $95 \% \mathrm{Cl}, 95 \%$ confident interval.

Table 7 Impact of secondary infection on clinical outcomes

\begin{tabular}{lcccc}
\hline Patient variable & ${ }^{a}$ Overall $(n=190)$ & No positive culture $(n=25)$ & Positive cultures $(n=165)$ & $P$ value \\
\hline Ventilator-free days in 28 days, mean (SD) & $2.6(6.2)(n=154)$ & $6.1(10.8)(n=15)$ & $2.2(5.4)(n=139)$ & 0.02 \\
28 days ICU mortality & $95(51.4)(n=185)$ & $12(50.0)(n=24)$ & $83(51.6)(n=161)$ & 0.89 \\
\hline
\end{tabular}

Data are presented as No. (\%) unless otherwise noted. ${ }^{a}$ Total sample size is 190 . Where data are unavailable, sample size for data is provided. SD, standard deviation; ICU, intensive care unit.

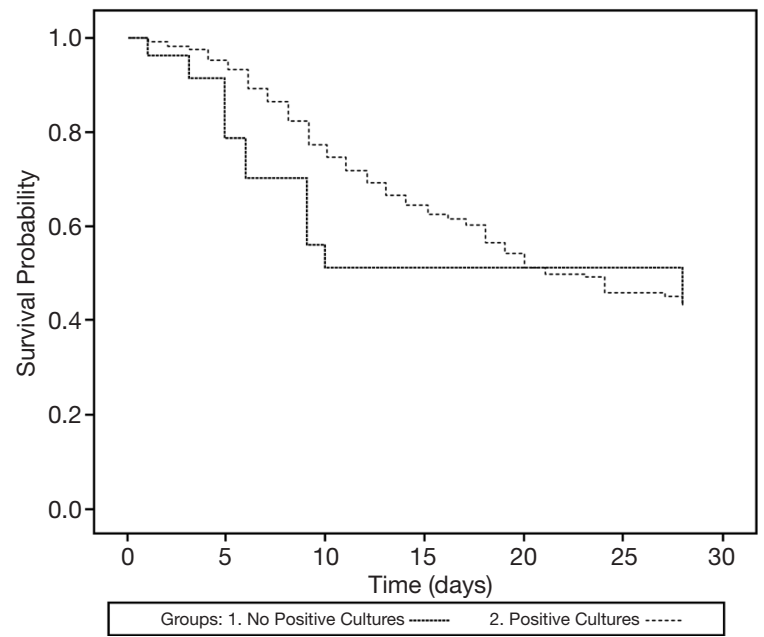

Figure 2 Twenty-eight-day ICU Kaplan-Meier survival curve comparing patients without positive cultures to those with positive cultures. $\mathrm{P}=0.46$.

and this positively correlated with the mortality rate (15). Even now, in the latest EPIC III study, the incidence of ICU acquired infection, which was with a substantial risk of in-hospital mortality, was $22 \%$ (16). In another study that focused on secondary infection in 2009 influenza A (H1N1), positive cultures were obtained in $38 \%$ of critically

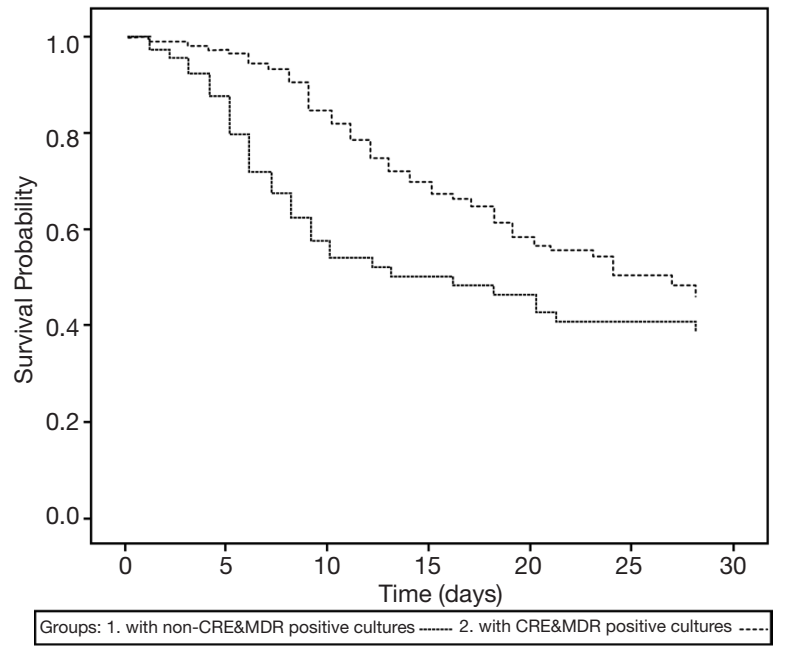

Figure 3 Twenty-eight-day ICU Kaplan-Meier survival curve comparing patients with carbapenem resistant Enterobacteriaceae (CRE) and multidrug resistant (MDR) positive cultures with those with non-CRE and MDR positive cultures. $\mathrm{P}=0.02$.

ill patients who were admitted to the ICU (6). However, the incidence of secondary infection in our study was surprisingly high $(86.6 \%)$. We thought the main reasons were as follows. First, although a complete nosocomial infection prevention and control system was set up in China 
according to the guidelines $(17,18)$, medical staff wore extensive personal protective equipment, and the heavy workload contributed to the incomplete implementation of these measures, especially during the early stage of the pandemic in Wuhan. It is worth noting that only one patient acquired bloodstream infection in The First Affiliated Hospital of Guangzhou Medical University; this was significantly less than the other two hospitals in Wuhan, which might be due to insufficient medical resources in Wuhan. This phenomenon was consistent with the previous opinion that practices to prevent health careacquired infections were generally absent in low-resource settings $(19,20)$. Second, SARS-CoV-2 induces severe lung damage, damages the respiratory tract epithelium, and causes apoptosis of lung macrophages and neutrophils, thus weakening barrier function (21), ultimately increasing susceptibility to secondary infection in the lung.

Similar to the EPIC III study (16), the majority of the organisms isolated in our study were gram negative bacteria, particularly $K$. pneumoniae and A. baumannii. Additionally, we found that the proportions of MDR bacteria and CRE were surprisingly high. CRE and MDR A. baumannii emerged as a major worldwide human health threat, as CRE infections are associated with high mortality and morbidity (22-24). In China, CRE and MDR $A$. baumannii represent a rapidly-emerging threat because the isolation rate has recently increased year by year (25), and this microbe reduces patients' clinical outcomes (26). Interestingly, our study found that these pathogens mainly occurred in Wuhan, and the proportions were much lower in Guangzhou. We considered the possibility that many ICUs were improvised in response to a sudden outbreak of the pandemic in Wuhan with a serious shortage of experienced ICU medical staff, and this was likely to be a major contributing factor to the spread of drug-resistant bacteria.

Our study demonstrated that advanced age and long duration of mechanical ventilation were independent high risk factors for secondary infection in severe and critical COVID-19 patients, consistent with previous studies and guidelines $(16,17)$. However, we failed to demonstrate that glucocorticoid and antibiotic usage were independently associated with the occurrence of secondary infections. The main reasons behind this may be that the limited sample size did not have sufficient power to evaluate the true effect and the proportions of glucocorticoid and antibiotic usage in patients both with and without positive cultures were high, which made it more difficult to assess the real effects.
In the EPIC III study, no specific organism was independently associated with a higher risk of death when considering all patients with secondary infections. Elderly age, higher simplified acute physiology score II, and comorbidities of metastatic cancer, HIV infection, and heart failure were independently associated with a higher risk of death. However, this variation was associated with patient- and disease-specific factors, with the process of care and intercountry differences. When considering only antibiotic-resistant organisms, infections with Klebsiella spp. resistant to $\beta$-lactam antibiotics (including third-generation cephalosporins and carbapenems), and carbapenemresistant Acinetobacter spp. were independently associated with an increased risk of death, highlighting the association of antibiotic resistance with mortality and the importance of good antibiotic stewardship (16). The results of our study also demonstrated that secondary infections decreased ventilator-free days at 28 days, and CRE and MDR bacterial infection decreased the 28-day ICU survival rate, consistent with previous studies (27-30).

Our study has some limitations. Firstly, this is a retrospective study. Selection bias may affect the results, further prospective studies are needed to assess the true incidence of secondary infection in COVID-19 patients. Secondly, the definition of secondary infection is based on the microbiological culture results. The clinical data may not be sufficient to differentiate between infection and colonization in a retrospective study, which may influence the interpretation of frequencies of "secondary infection". However, the result of this study is still reasonably 'representative' and reflected the real situation during the early stage of the COVID-19.

\section{Conclusions}

In a retrospective cohort of severe and critical COVID-19 patients admitted to ICUs at the early stage of the COVID-19 pandemic in China, the prevalence of secondary infection was high, particularly CRE and MDR bacteria, resulting in poor clinical outcomes. Prevalence of secondary infection in COVID-19 patients may vary across different regions, which reveal matching of demand and supply of healthcare resources affect the prevalence of secondary infection.

\section{Acknowledgments}

Funding: This work was supported by Emergency Key 
Program of Guangzhou Laboratory [Grant No. EKPG2117], The Natural Science Foundation of Guangdong Province, China [2020A1515011459], The Major special projects of the Ministry of Science and Technology [2020YFC0841300] and National Natural Science Foundation of China [81970071 and 82070084].

\section{Footnote}

Reporting Checklist: The authors have completed the STROBE reporting checklist. Available at https://dx.doi. org/10.21037/apm-21-833

Data Sharing Statement: Available at https://dx.doi. org/10.21037/apm-21-833

Peer Review File: Available at https://dx.doi.org/10.21037/ apm-21-833

Conflicts of Interest: All authors have completed the ICMJE uniform disclosure form (available at https://dx.doi. org/10.21037/apm-21-833). The authors have no conflicts of interest to declare.

Ethical Statement: The authors are accountable for all aspects of the work in ensuring that questions related to the accuracy or integrity of any part of the work are appropriately investigated and resolved. The study was conducted in accordance with the Declaration of Helsinki (as revised in 2013). This study was approved separately by the Independence Ethics Committee of Union Hospital, Tongji Medical College, Huazhong University of Science and Technology (Approval No.: 2020-0307), Medical Ethics Committee of Wuhan Infectious Disease Hospital (Approval No.: KY-2020-56.01) and Ethics Committee of the First Affiliated Hospital of Guangzhou Medical University (2020065). Informed consent was waived due to the retrospective nature of the study.

Open Access Statement: This is an Open Access article distributed in accordance with the Creative Commons Attribution-NonCommercial-NoDerivs 4.0 International License (CC BY-NC-ND 4.0), which permits the noncommercial replication and distribution of the article with the strict proviso that no changes or edits are made and the original work is properly cited (including links to both the formal publication through the relevant DOI and the license). See: https://creativecommons.org/licenses/by-nc-nd/4.0/.

\section{References}

1. WHO Coronavirus (COVID-19) Dashboard. Available online: https:// https://covid19.who.int/. Accessed on 31 March 2021.

2. Richardson S, Hirsch JS, Narasimhan M, et al. Presenting Characteristics, Comorbidities, and Outcomes Among 5700 Patients Hospitalized With COVID-19 in the New York City Area. JAMA 2020;323:2052-9.

3. Zhou F, Yu T, Du R, et al. Clinical course and risk factors for mortality of adult inpatients with COVID-19 in Wuhan, China: a retrospective cohort study. Lancet 2020;395:1054-62.

4. Yang X, Yu Y, Xu J, et al. Clinical course and outcomes of critically ill patients with SARS-CoV-2 pneumonia in Wuhan, China: a single-centered, retrospective, observational study. Lancet Respir Med 2020;8:475-81.

5. MacIntyre CR, Chughtai AA, Barnes M, et al. The role of pneumonia and secondary bacterial infection in fatal and serious outcomes of pandemic influenza a(H1N1)pdm09. BMC Infect Dis 2018;18:637.

6. Muscedere J, Ofner M, Kumar A, et al. The occurrence and impact of bacterial organisms complicating critical care illness associated with 2009 influenza A(H1N1) infection. Chest 2013;144:39-47.

7. Yap FH, Gomersall CD, Fung KS, et al. Increase in methicillin-resistant Staphylococcus aureus acquisition rate and change in pathogen pattern associated with an outbreak of severe acute respiratory syndrome. Clin Infect Dis 2004;39:511-6.

8. Shang Y, Pan C, Yang X, et al. Management of critically ill patients with COVID-19 in ICU: statement from frontline intensive care experts in Wuhan, China. Ann Intensive Care 2020;10:73.

9. Wang D, Hu B, Hu C, et al. Clinical Characteristics of 138 Hospitalized Patients With 2019 Novel CoronavirusInfected Pneumonia in Wuhan, China. JAMA 2020;323:1061-9.

10. Wu C, Chen X, Cai Y, et al. Risk Factors Associated With Acute Respiratory Distress Syndrome and Death in Patients With Coronavirus Disease 2019 Pneumonia in Wuhan, China. JAMA Intern Med 2020;180:934-43.

11. Diagnosis and Treatment protocol for COVID-19 (Trial Version 7) by National Health Commission \& State Administration of Traditional Chinese Medicine. Available online: http://www.nhc.gov.cn/yzygj/s7653p/202003/46c9 294a7dfe4cef80dc7f5912eb1989/files/ce3e6945832a438eaa e415350a8ce964.pdf. Accessed on 4 March 2020. 
12. Chen X, Liao B, Cheng L, et al. The microbial coinfection in COVID-19. Appl Microbiol Biotechnol 2020;104:7777-85.

13. Søgaard KK, Baettig V, Osthoff M, et al. Communityacquired and hospital-acquired respiratory tract infection and bloodstream infection in patients hospitalized with COVID-19 pneumonia. J Intensive Care 2021;9:10.

14. Thomsen K, Pedersen HP, Iversen S, et al. Extensive microbiological respiratory tract specimen characterization in critically ill COVID-19 patients. APMIS 2021;129:431-7.

15. Vincent JL, Bihari DJ, Suter PM, et al. The prevalence of nosocomial infection in intensive care units in Europe. Results of the European Prevalence of Infection in Intensive Care (EPIC) Study. EPIC International Advisory Committee. JAMA 1995;274:639-44.

16. Vincent JL, Sakr Y, Singer M, et al. Prevalence and Outcomes of Infection Among Patients in Intensive Care Units in 2017. JAMA 2020;323:1478-87.

17. Kalil AC, Metersky ML, Klompas M, et al. Management of Adults With Hospital-acquired and Ventilator-associated Pneumonia: 2016 Clinical Practice Guidelines by the Infectious Diseases Society of America and the American Thoracic Society. Clin Infect Dis 2016;63:e61-e111.

18. Mermel LA, Allon M, Bouza E, et al. Clinical practice guidelines for the diagnosis and management of intravascular catheter-related infection: 2009 Update by the Infectious Diseases Society of America. Clin Infect Dis 2009;49:1-45.

19. Bebell LM, Muiru AN. Antibiotic use and emerging resistance: how can resource-limited countries turn the tide? Glob Heart 2014;9:347-58.

20. Ombelet S, Ronat JB, Walsh T, et al. Clinical bacteriology in low-resource settings: today's solutions. Lancet Infect Dis 2018;18:e248-58.

21. Xu Z, Shi L, Wang Y, et al. Pathological findings of

Cite this article as: Sang L, Xi Y, Lin Z, Pan Y, Song B, Li CA, Zheng X, Zhong M, Jiang L, Pan C, Zhang W, Lv Z, Xia J, Chen N, Wu W, Xu Y, Chen S, Liu D, Liang W, Liu X, Liu X, Li S, Zhong N, Ye D, Xu Y, Zhang N, Zhang D, Li Y. Secondary infection in severe and critical COVID-19 patients in China: a multicenter retrospective study. Ann Palliat Med 2021;10(8):8557-8570. doi: 10.21037/apm-21-833
COVID-19 associated with acute respiratory distress syndrome. Lancet Respir Med 2020;8:420-2.

22. Xu L, Sun X, Ma X. Systematic review and meta-analysis of mortality of patients infected with carbapenem-resistant Klebsiella pneumoniae. Ann Clin Microbiol Antimicrob 2017;16:18.

23. Butler DA, Biagi M, Tan X, et al. Multidrug Resistant Acinetobacter baumannii: Resistance by Any Other Name Would Still be Hard to Treat. Curr Infect Dis Rep 2019;21:46.

24. Gomez-Simmonds A, Uhlemann AC. Clinical Implications of Genomic Adaptation and Evolution of Carbapenem-Resistant Klebsiella pneumoniae. J Infect Dis 2017;215:S18-27.

25. Hu F, Guo Y, Zhu D, et al. CHINET 2016 surveillance of bacterial resistance in China. Chin J Infect Chemother 2017;17:481-91.

26. Huang W, Qiao F, Zhang Y, et al. In-hospital Medical Costs of Infections Caused by Carbapenem-resistant Klebsiella pneumoniae. Clin Infect Dis 2018;67:S225-30.

27. Lee NY, Lee HC, Ko NY, et al. Clinical and economic impact of multidrug resistance in nosocomial Acinetobacter baumannii bacteremia. Infect Control Hosp Epidemiol 2007;28:713-9.

28. Jang TN, Lee SH, Huang CH, et al. Risk factors and impact of nosocomial Acinetobacter baumannii bloodstream infections in the adult intensive care unit: a case-control study. J Hosp Infect 2009;73:143-50.

29. Asim P, Naik NA, Muralidhar V, et al. Clinical and economic outcomes of Acinetobacter vis a vis nonAcinetobacter infections in an Indian teaching hospital. Perspect Clin Res 2016;7:28-31.

30. Sunenshine RH, Wright MO, Maragakis LL, et al. Multidrug-resistant Acinetobacter infection mortality rate and length of hospitalization. Emerg Infect Dis 2007;13:97-103. 